\title{
Vitalidade Subjetiva, Sentido na Vida e Religiosidade em Idosos: Um Estudo Correlacional
}

\author{
Danielly Costa Roque Vieira \\ Universidade Federal da Paraíba, João Pessoa, PB, Brasil \\ Thiago Antonio Avellar de Aquino ${ }^{1}$ \\ Departamento de Ciências das Religiões da Universidade Federal da Paraíba, \\ João Pessoa, PB, Brasil
}

\begin{abstract}
Resumo
O presente artigo tem como objetivo identificar as relações entre a religiosidade, a percepção de sentido na vida, a percepção ontológica do tempo e a vitalidade subjetiva de pessoas idosas. O estudo contou com 100 idosos que participavam de grupos de terceira idade com idade média de 67,2 anos $(D P=5,6)$, que responderam à Escala de Atitudes Religiosas (EAR), Questionário de Sentido de Vida (QSV), Escala de Percepção Ontológica do Tempo (EPOT) e a Escala de Vitalidade Subjetiva (EVS) e perguntas demográficas. Constatou-se que a Vitalidade Subjetiva correlacionou-se diretamente com a presença de sentido, o passado, o presente e o futuro. Já a busca de sentido associou-se diretamente com o comportamento religioso e o sentimento religioso; enquanto a presença de sentido o fez com conhecimento religioso, comportamento religioso, percepção do presente e percepção do futuro. Os resultados foram discutidos fundamentados na concepção da Logoterapia e Análise Existencial.
\end{abstract}

Palavras-chave: Envelhecimento, religiosidade, sentido na vida, vitalidade.

\section{Subjective Vitality, Meaning in Life and Religiosity in Older People: A Correlational Study}

\begin{abstract}
This article aims to identify the relationship between the religiosity, the perception of meaning in life, the ontological perception of time, and the subjective vitality in elder people. This research included a sample of 100 senior citizens that used to attend senior citizens groups, with an average age 67.2 years old $(S D=5.6)$, who responded to the Religious Attitudes Scale (RAS-20), the Meaning in Life Questionnaire (MLQ), the Ontological Perception of Time Scale (OPTS), the Subjective Vitality Scale (SVS), as well as answered a demography questionary. The results showed a positive correlation between the Subjective Vitality with presence for meaning, as well as to the individual past, present, and future. On the other hand, the research shows that the Search for Meaning is closely related to the religious behavior and to religious feeling. Moreover, the research shows that the presence of meaning is related to the knowledge religious, behavior, as well as to the perception of present and future. These findings were discussed based on the conception of Logotherapy and Analysis Existential.
\end{abstract}

Keywords: Aging, religiosity, meaning in life, vitality.

Endereço para correspondência: Rua Mário Batista Júnior, 75, Apto. 301, Miramar, João Pessoa, PB, Brasil 58043-130. Fone: (83) 3507-3768. E-mail: logosvitae@hotmail.com, thiago.aquino@pesquisador.cnpq.br Apoio financeiro: Conselho Nacional de Desenvolvimento Científico e Tecnológico (CNPq). 


\section{Vitalidad Subjetiva, Sentido en la Vida y Religiosidad en los Ancianos: Un Estudio Correlacional}

\section{Resumen}

Este artículo tiene el objetivo de identificar las relaciones entre la religiosidad, la percepción del sentido de la vida, percepción ontológica del tiempo y vitalidad subjetiva en adultos mayores. Participaron 100 ancianos con una edad promedio de 67.2 años $(S D=5.6)$ que contestaron la Escala de Actitudes Religiosas (EAR), el Cuestionario Sentido en la Vida (CSL), la Escala de Percepción Ontológico del Tiempo (EPOT), la Escala de Vitalidad Subjetiva (EVS), y preguntas demográficas. Los resultados alcanzados mostraron que la vitalidad subjetiva se correlacionó directamente con la presencia de sentido, el pasado, el presente y el futuro. La búsqueda de sentido se asoció directamente con el comportamiento y sentimiento religioso; mientras que la presencia de significado lo hizo con el conocimiento religioso, el comportamiento religioso, la percepción del futuro y el presente. Los resultados fueron discutidos en función de las concepciones de la Logoterapia y Análisis Existencial.

Palabras clave: Envejecimiento, religiosidad, sentido en la vida, vitalidad.

Segundo a Organização Mundial de Saúde (World Health Organization [WHO]), a população acima de 60 anos vem crescendo nas últimas décadas (2002). Concomitantemente, observa-se que muitos estudos vêm abordando temáticas nessa fase do desenvolvimento humano. As pesquisas no contexto de envelhecimento abordam diversas temáticas, tais como: a relação entre religião e satisfação com a vida (Dendena, Dallazen, Lyra, \& Tosi, 2011), religiosidade e qualidade de vida em instituições asilares (Carneiro, 2009), religiosidade e satisfação com a vida/bem-estar (Araújo et al., 2008; Duarte, Lebrão, Tuono, \& Laurenti, 2008), resiliência e espiritualidade (Silva \& Alves, 2007), espiritualidade e percepção do sentido de vida da velhice tardia (Freitas, 2010), prevenção e promoção de saúde (Leandro-França \& Murta, 2014) e saúde mental e religiosidade/espiritualidade (Parker et al., 2003).

Contudo, ainda resta saber em que medida o sentido da vida compartilha variância com a vitalidade subjetiva, sobretudo em pessoas idosas. Tal empreendimento justifica-se na medida em que a Organização Mundial de Saúde vem adotando o termo envelhecimento ativo (active ageing) para designar o processo de otimização e qualidade de vida no envelhecimento humano (WHO, 2002). Dessa forma, compreende-se que a vitalidade possa ser relevante para a autono- mia, independência, qualidade de vida e expectativa de vida saudável no idoso.

\section{Vitalidade Subjetiva}

A palavra vitalidade deriva-se do latim, $v i$ talitate, cuja raiz etiológica significa vida (Menezes, 2012). A ideia de vitalidade também se faz presente na medida de bem estar subjetivo, e a sua significação pode mudar de indivíduo para indivíduo (Ryan \& Deci, 2001). Indubitavelmente, sua conceituação refere-se a uma dimensão positiva da saúde, posto que pode ser classificada como parte do construto bem-estar afetivo, compreendendo assim um afeto positivo que sugere a experiência de se estar vivo (Nix, Ryan, Manly, \& Deci, 1999; Paschoal \& Tamayo, 2008).

Ryan e Frederick (1997) definiram a vitalidade subjetiva em termos de um sentimento de vivacidade e de uma sensação de energia pessoal. Estudos empíricos sugerem que essa variável tem se correlacionado com autorrealização, autoestima (Ryan \& Frederick, 1997) e satisfação com a vida (Gouveia et al., 2012). Ademais, compreende-se que a vitalidade pode ser consumida ou gasta, mas também pode ser mantida ou ampliada (Ryan \& Frederick, 1997). Nessa direção, julga-se que, possivelmente, a percepção do sentido na vida e a espiritualidade desempenham um papel importante na manutenção da vitalidade subjetiva. 


\section{Espiritualidade como Busca de Sentido na Vida}

De forma geral, a espiritualidade pode ser definida como uma busca de sentido com ou sem a adesão a uma religião específica (Mowat \& O’Neill, 2013). Nessa perspectiva, Frankl (1969/2011) inaugura um sistema de pensamento denominado de Logoterapia, que pode ser mais bem compreendido como uma escola de psicoterapia centrada no sentido da vida. O pressuposto fundamental é que o ser humano é o único ente que questiona acerca do sentido de sua vida tendo em conta que possui uma espiritualidade, por vezes chamada de dimensão noológica, de que se derivam os fenômenos genuinamente humanos (intencionalidade, preocupação com valores e sentidos, dentre outros). A partir dessa dimensão o indivíduo pode se constituir como um ser que transcende os seus condicionamentos e busca realizar valores que possam se constituir em razões para viver.

De acordo com Frankl (1946/1989, 1946/ 2005a), a motivação primária do ser humano é a procura de um significado para a vida. Essa busca ocasionaria uma tensão interior, que consiste em um prerrequisito indispensável para a saúde mental, posto que "a saúde mental está baseada em certo grau de tensão, tensão entre aquilo que já se alcançou e aquilo que deveria alcançar, ou o hiato entre o que se é e o que deveria vir a ser" (Frankl, 1969/2011, p. 61). O sentido pode ser logrado por meio de três valores: vivenciais (vivenciar algo ou alguém), criativos (criar algo como trabalhos e ações no mundo) e atitudinais (tomar uma postura perante o sofrimento inevitável). Segundo Frankl (1969/2011), os valores se encontram no mundo, partindo do pressuposto de que é no mundo que o ser humano deve descobrir e preencher os sentidos.

No que se refere aos estudos empíricos, Oliveira e Silva (2013) realizaram um estudo junto a 146 idosos encontrando associações positivas entre a realização de sentido e o bem-estar psicológico $(r=0,55, p<0,001)$. Além de se relacionar com a religiosidade (Aquino et al., 2009), o construto sentido da vida se associa também com indicadores de bem-estar, tais como: satisfação com a vida, afetos positivos e saúde men- tal (King, Hicks, \& Del Gaiso, 2006; Mascaro, 2006; Mascaro \& Rosen, 2008).

De forma geral, compreende-se que o sentido da vida encontra-se nas relações do ser humano com o mundo, diferindo de pessoa para pessoa e de situação para situação. Esse pressuposto parte de um fundamento antropológico e existencial no qual não se pode viver de forma verdadeiramente humana sem uma perspectiva de um sentido para a vida. Entretanto, a vontade de sentido pode ser frustrada e ocasionar uma sensação de falta de conteúdo na vida ou um vazio/ frustração existencial. Essa sensação pode ocasionar o que ele define como neurose noogênica, posto que teria sua etiologia na dimensão noológica da existência humana. Esta, por sua vez, conforme a Logoterapia, pode surgir decorrente de problemas existenciais, conflitos valorativos, ou remorso de consciência (Frankl, 1969/2011).

Segundo Kimble (2009), o que contribui para a crise de sentido na sociedade moderna seria a falta dos símbolos e dos ritos de passagens em torno do ciclo da vida. Ramovs (2009) destaca que para cada estágio da vida há uma tarefa social e um sentido específico. Por exemplo, a juventude teria uma tarefa de se preparar para a constituição de uma família e o ingresso no mundo do trabalho; a vida adulta seria um período de produtividade, e a terceira fase, da aposentadoria até a morte, o período de maturidade e sabedoria. Esse mesmo autor compreende que as três gerações são complementares, dessa forma, o vazio existencial origina-se no momento em que a conexão entre as gerações é enfraquecida.

\section{Temporalidade e Sentido da Vida no Idoso}

Considerando que a temporalidade se relaciona com a busca de sentido (Frank1, 1978/ 2005b), torna-se fundamental compreender a visão da Logoterapia sobre essa temática. Segundo esta teoria, o ser humano está imbuído de seu passado (realidades vivenciadas) e seu futuro (possibilidade de vir a ser), entre o ser e o não ser, entre o que já realizou e o que ainda deve realizar (Aquino, 2013). Na compreensão de Pintos (2014), o homem é um ser temporal, quer seja na perspectiva do Kronos (tempo cro- 
nológico: passado, presente e futuro), quer seja na vivência do Kairós (dimensão mais pessoal e vivencial do tempo).

Para Pintos (1992), o homem caracteriza-se pela sua capacidade de se relacionar com o tempo em suas demais dimensões, de tal forma que ele possa se projetar no futuro, viver o presente em plenitude, reviver imagens do passado, assim como reconhecê-los e localizá-los no tempo e no espaço. Frankl (1969/2011) compreende que o passado não é apenas real como também perene. O ser humano relaciona-se com o passado por meio das suas reminiscências e, indubitavelmente, questiona-se acerca do sentido de seus dias (Pintos, 1992).

O ser humano possui no seu "ser passado" as possibilidades que já foram transformadas em realidade. Dessa forma, quando um valor, em um dado momento, é logrado no mundo por um indivíduo, este se torna concreto e real, enquanto as possibilidades que ainda não foram realizadas são passíveis de serem perdidas (Frankl, 1978/2005b; Lukas, 1992). Por esse motivo, Lukas (1992) considera relevante um balanço existencial, sobretudo em pessoas idosas, a partir das três perspectivas da temporalidade: $O$ olhar para trás, a tarefa do presente, e o olhar para frente.

$O$ olhar para trás consiste em avaliar tudo o que foi vivido e sofrido, tanto a percepção dos êxitos quanto dos fracassos. Os êxitos resumem-se naquilo que foi logrado com dignidade, o que resulta em uma sensação de dever. Por outro lado, tanto as possibilidades que não foram concretizadas quanto os fracassos, também se constituem como o foco perceptivo na vida de uma pessoa idosa.

A tarefa do presente consiste em avaliar as perdas e encontrar novas adaptações de atividades adequadas à própria situação, já que na velhice as tarefas a serem cumpridas são escassas. Não obstante, o olhar para frente, torna-se imperativo, considerando que o idoso ainda possui possibilidades de vir-a-ser, mesmo que essas possibilidades se encontrem mais restritas quando comparadas ao período da juventude (Lukas, 1992).

Grande parte dos idosos utiliza a religiosidade como estratégias de enfrentamento em situações estressantes e como uma forma de encontrar sentido (Ardelt \& Koenig, 2007; Koenig, George, \& Siegler, 1988). Autores como Maugans (1996) defendem que a espiritualidade pode ser compreendida como um sistema de crenças que proporciona vitalidade e sentido para a vida. Conforme Duarte et al. (2008), a religiosidade e a espiritualidade podem contribuir para o bem-estar, além de reduzir os níveis de depressão, morbidade, angústia e mortalidade. Nesse sentido, a religiosidade pode dar uma resposta plausível para as questões existenciais dos idosos na medida em que a religião fornecer um sistema de significado para a vida, para o sofrimento e para a morte (Goldstein \& Sommerhalder, 2002). Tendo em conta a relevância das questões existenciais no processo de envelhecimento, o objetivo do presente estudo foi conhecer em que medida a percepção do sentido de vida e a atitude religiosa se relacionam com a vitalidade subjetiva em uma amostra de pessoas idosas.

\section{Método}

\section{Participantes}

Participaram desse estudo 100 idosos que frequentavam grupos de convivência localizados nas cidades de Santa Rita (PB) e João Pessoa (PB). A maioria do sexo feminino (90\%), com a idade média de 67,1 $(D P=5,6)$ e amplitude de 60 a 87 anos. Quanto ao grau de escolaridade, a maioria possuía o ensino fundamental incompleto $(64,0 \%)$. No que se refere ao estado civil, observou-se que $54 \%$ eram casados, $40 \%$ eram viúvos, e $6 \%$ divorciados. Com relação à afiliação religiosa, $80 \%$ afirmaram que eram católicos e $20 \%$, evangélicos.

\section{Instrumentos}

Escala de Vitalidade Subjetiva (EVS). Esta escala foi originalmente criada por Ryan e Frederick (1997). A sua versão final contém sete itens (e.g. tenho energia e disposição; sinto-me vivo e cheio de vitalidade) dispostos em uma escala de sete pontos variando de 1 = Nada Verdadeiro a $7=$ Totalmente Verdadeiro. Gouveia et al. (2012) adaptaram e validaram esse instrumento 
para o contexto brasileiro confirmando sua solução unifatorial com o índice de alfa de Cronbach de 0,73 .

Questionário de Sentido de Vida (QSV). Este questionário foi proposto por Steger, Frazier, Oishi e Kaler (2006). Em sua versão original contém 10 itens para ser avaliado numa escala de 7 pontos, sendo $1=$ totalmente falso e $7=$ totalmente verdadeiro. Damásio (2013) realizou uma adaptação dessa medida para o contexto brasileiro constatando a presença de dois fatores: fator presença de sentido, com alfa de Cronbach igual a 0,88 (ex.: eu compreendo o sentido da minha vida; minha vida tem um sentido claro; eu tenho uma boa consciência do que faz minha vida ter sentido; eu descobri um sentido de vida satisfatório) e o fator busca de sentido, com alfa de Cronbach igual a 0,85 (ex.: eu estou procurando alguma coisa que faça com que minha vida tenha sentido; eu sempre estou em busca do sentido da minha vida).

Escala de Percepção Ontológica do Tempo (EPOT). Foi elaborado por Aquino (2009). É constituída por um conjunto de dez itens, sendo que três estão relacionados com uma perspectiva do tempo: passado $(\alpha=0,64$; e.g. sinto-me realizado com o que alcancei); presente ( $\alpha$ $=0,74$; e.g. vejo sempre um motivo para estar no mundo) e futuro $(\alpha=0,73$; e.g. vejo muitas possibilidades de escolha). Um último item visa identificar a satisfação geral com o "eu" ao longo do tempo (tenho que admitir que há uma grande distância entre quem eu sou e quem eu poderia ser). Todos os itens são dispostos numa escala de cinco pontos: $1=$ discordo totalmente e $5=$ concordo totalmente.

Escala de Atitudes Religiosas (EAR-20). Instrumento elaborado por Aquino, Gouveia, Silva e Aguiar (2013), originalmente em português, composto por vinte itens e quatro fatores: Comportamento religioso $(\alpha=0,82$, e.g. Frequento as celebrações de minha religião); conhecimento religioso $(\alpha=0,85$, e.g. Procuro conhecer as doutrinas ou preceitos da minha religião); sentimento religioso $(\alpha=0,65$, e.g. Extravaso a tristeza ou alegrias através de músicas religiosas); e corporeidade religiosa ( $\alpha=0,90$, e.g., Ajoelho-me para fazer minha oração pesso- al com Deus). Todos os itens são dispostos numa escala intervalar, com os seguintes extremos: 1 (Nunca) e 5 (Sempre).

Questionário Sociodemográfico. Objetivou exclusivamente descrever os participantes da pesquisa por meio dos seguintes indicadores: idade, sexo, estado civil, escolaridade e religião.

\section{Procedimentos}

Inicialmente esse estudo foi aprovado pelo Comitê de Ética em Pesquisa (CEP) com seres humanos do Hospital Universitário Lauro Wanderley, da Universidade Federal da Paraíba. Todos os participantes assinaram um termo de consentimento livre e esclarecido (TCLE), como condição necessária, e foram alertados que a participação seria voluntária e que a qualquer momento poderiam desistir sem nenhuma penalidade. Enfatizou-se também o caráter anônimo das entrevistas e que ninguém precisaria se identificar posto que as respostas seriam analisadas coletivamente, seguindo todas as recomendações da Resolução ${ }^{\circ}$ 466/12, do Conselho Nacional de Saúde (2012). Os instrumentos que estavam dispostos em um caderno de resposta foram administrados individualmente pelo primeiro pesquisador nos centros de convivência dos idosos, com uma duração média de 40 minutos.

\section{Procedimentos para Análise dos Dados}

Os dados foram analisados com o PASW (versão 17.0), com o qual realizaram-se análise fatorial no conjunto de itens da escala de Vitalidade Subjetiva bem como o cálculo de consistência interna por meio do alfa de Cronbach. Por fim foi realizado o teste de correlação de Pearson com o intuito de averiguar as associações entre as variáveis em estudo.

\section{Resultados}

\section{Evidências de Validade Fatorial da Escala de Vitalidade Subjetiva para Idosos}

Antes de examinar a fatorabilidade da matriz de correlações, foi verificada a pertinência em realizar essa análise. Os resultados obtidos 
apontam a conveniência da realização da análise fatorial: $\mathrm{KMO}=0,85$, Teste de Esfericidade de Bartlett, $\chi^{2}=241,27 ; p \leq 0,001$ (Tabachnick \& Fidell, 2001). Em seguida, foi efetuada uma análise de PAF (principal axis fatoring), sem fixar o número de fatores a serem extraídos; a análise sugeriu a presença de um único fator com valor próprio (eigenvalue) de 3,46, explicando 49,5\% da variância total. Esta descrição detalhada poderá ser observada na Tabela 1. Destarte, pode-se considerar que a escala de Vitalidade Subjetiva adequa-se melhor a uma estrutura unifatorial considerando que tanto o critério de Catell (cf. Figura 1) quanto o critério de Kaiser (escolha dos fatores com valores próprios superiores a 1), corroboram essa estrutura.

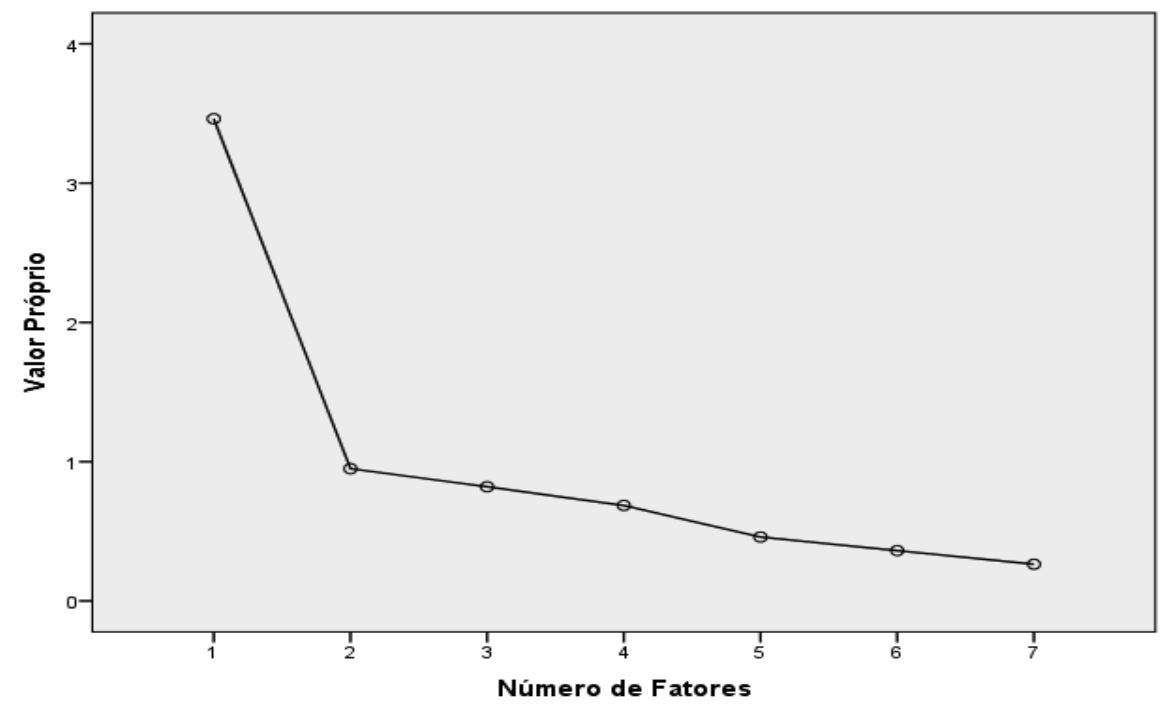

Figura 1. Representação gráfica dos valores próprios da Escala de Vitalidade Subjetiva.

Tabela 1

Estrutura Fatorial da Escala de Vitalidade Subjetiva

\begin{tabular}{lc}
\hline \multicolumn{1}{c}{ Itens } & Carga Fatorial \\
\hline Quase sempre me sinto disposto e ativo & $0,85^{*}$ \\
Sinto-me vitalizado & $0,78^{*}$ \\
Sinto-me vivo e cheio de vitalidade & $0,77^{*}$ \\
Tenho energia e disposição & $0,75^{*}$ \\
Algumas vezes me sinto tão vivo a ponto de transbordar & $0,53^{*}$ \\
Desejo viver cada novo dia & $0,42^{*}$ \\
Não me sinto muito disposto & $-0,26$ \\
Valor próprio & 3,45 \\
\% Variância Explicada & 49,5 \\
Alfa de Cronbach & 0,84 \\
\hline
\end{tabular}

Nota. $* \geq 0,40$ (Carga fatorial considerada satisfatória).

A Tabela 1 descreve as cargas fatoriais dos itens da Escala de Vitalidade Subjetiva. Observa-se que as saturações iguais ou superiores a 0,42 constituíram uma estrutura unifatorial a qual pode ser conceituada como vitalidade. A menor saturação ocorreu no item Desejo viver a cada 
novo dia $(0,42)$, enquanto a maior saturação sobreveio no item Quase sempre me sinto disposto e ativo $(0,85)$. A consistência interna deste fator, calculada por meio do Alfa de Cronbach $(\alpha)$, obteve o índice de 0,84 , o que foi considerado um parâmetro satisfatório.

\section{Correlatos Existenciais da Vitalidade Subjetiva}

Considerando que a escala de Vitalidade Subjetiva apresentou índices psicométricos satisfatórios para a amostra em questão, verificou-se a associação entre variáveis do estudo como pode ser constatado por meio da matriz correlacional (Tabela 2).

\section{Tabela 2}

Matriz da Correlação entre Fatores de Medidas da Escala Vitalidade Subjetiva (EVS) e Escala de Percepção Ontológica do Tempo (EPOT) e QSV e Atitude Religiosa

\begin{tabular}{lccccccccc}
\hline Indicadores existenciais & 1 & 2 & 3 & 4 & 5 & 6 & 7 & 8 & 9 \\
\hline 1. Vitalidade & - & & & & & & & & \\
2. Presença de sentido & $0,20^{*}$ & & & & & & & & \\
3. Busca de sentido & $-0,14$ & 0,04 & & & & & & & \\
4. Conhecimento Religioso & 0,08 & $0,24^{*}$ & 0,10 & & & & & & \\
5. Comportamento Religioso & 0,08 & $0,21^{*}$ & $0,24^{*}$ & $0,61^{* * *}$ & & & & & \\
6. Sentimento Religioso & 0,06 & 0,16 & $0,38^{* * *}$ & $0,32^{* *}$ & $0,43^{* * *}$ & & & & \\
7. Corporeidade Religiosa & 0,02 & $-0,08$ & 0,06 & $0,33^{* *}$ & $0,41^{* * *}$ & $0,44^{* * *}$ & & & \\
8. Percepção do Passado & $0,38^{* * *}$ & 0,12 & $-0,15$ & $-0,09$ & $-0,04$ & 0,02 & 0,13 & & \\
9. Percepção do Presente & $0,48^{* * *}$ & $0,29^{* *}$ & $-0,05$ & 0,03 & 0,10 & 0,12 & 0,08 & $0,55^{* * *}$ & \\
10. Percepção do Futuro & $0,43^{* * *}$ & $0,26^{* *}$ & 0,11 & 0,12 & 0,11 & 0,13 & 0,04 & 0,18 & $0,41^{* * *}$ \\
\hline
\end{tabular}

${ }^{*} p<0,05 ; * * p<0,01 ; * * * p<0,0001$.

Constata-se que a Vitalidade Subjetiva correlacionou-se diretamente com as seguintes variáveis: presença de sentido $(r=0,20 ; p<$ $0,05)$, passado $(r=0,38 ; p<0,0001)$, presente $(r=0,48 ; p<0,0001)$ e futuro $(r=0,43 ; p<$ $0,0001)$. Já a busca de sentido associou-se diretamente com o comportamento religioso $(r=0,24, p<0,05)$ e o sentimento religioso $(r=0,38, p<0,0001)$; enquanto a presença de sentido o fez com conhecimento religioso $(r=$ $0,24, p<0,05)$, comportamento religioso $(r=$ $0,21, p<0,05)$, percepção do presente $(r=29, p$ $<0,01)$ e percepção do futuro $(r=26, p<0,01)$.

\section{Discussão}

O presente estudo buscou conhecer as relações entre religiosidade, sentido de vida e vitalidade subjetiva em idosos. Embora se admita que os objetivos foram plenamente atingidos, torna-se necessário apresentar algumas limitações desse estudo. No que diz respeito à amostra, esta foi constituída por conveniência e não por meio de um procedimento aleatório. Dessa forma, não pode ser considerada como representativa da população estudada. Nesse sentido os resultados aqui apontados representam tão somente o perfil da amostra estudada, não sendo passíveis de generalização.

\section{Estrutura Fatorial e Consistência Interna da Escala de Vitalidade Subjetiva}

Sobre a adaptação da Escala de Vitalidade Subjetiva para idosos, essa se apresentou adequada, sugerindo evidências de validade de construto. Ademais, também apresenta evidência de precisão com índice superiore ao que é 
sugerido pela literatura: 0,70 (Nunnally, 1991; Pasquali, 2003). O modelo aqui proposto para aferir a Vitalidade Subjetiva em idosos excluiu o item não me sinto muito disposto, o que também é corroborado pelos estudos de Bostic, Rubio e Hood (2000) e Gouveia et al. (2012), na medida em que os mesmos sugerem uma melhor adequação quando eliminam o referido item.

\section{Religiosidade e Sentido da Vida}

De forma geral, as medidas de religiosidade se associam diretamente com o sentido da vida (Aquino et al., 2009). No presente estudo, duas subscalas correlacionam-se positivamente com a presença de sentido: Conhecimento e Comportamento Religioso. Já a busca de sentido associou-se com o comportamento religioso e o sentimento religioso. A religiosidade pode ajudar no enfrentamento de questões existenciais, seja na busca de um sentido para a vida em decorrência de uma maior consciência da finitude e da morte, ou para aumentar a sensação de sentido considerando que a religiosidade pode influenciar na percepção de um sentido.

Esse resultado pode ser mais bem compreendido à luz da teoria de Frankl (1946/1989) quando a mesma compreende que o homem antigo encontrava mais facilmente o sentido por meio da tradição. De fato, a religiosidade pode ser concebida como uma fonte de sentido para a vida humana, na medida em que procura responder as inquietações existenciais referentes à finitude humana.

A tradição, juntamente com as normas e prescrições, já apontavam, a priori, o que seria o sentido. Dessa forma, os idosos que foram entrevistados apresentam mais uma sensação de sentido na medida em que fazem leituras religiosas e se comportam mediante os ensinamentos de sua religião. Esses resultados são corroborados com o estudo de Ardelt (2003) e Steger et al. (2006) quando observaram uma associação positiva entre religiosidade intrínseca e a presença de sentido. Outros estudos também sugerem que pessoas mais religiosas apresentam uma maior sensação de sentido na vida (Steger \& Frazier, 2005).

Guimarães e Avezum (2007) averiguaram em seus estudos que maiores níveis de envolvi- mento religioso estão associados positivamente a indicadores de satisfação com a vida, felicidade e afetos positivos. Frankl (2007) compreende a religiosidade a partir de uma conotação pessoal, na qual só o indivíduo pode se decidir por Deus, atribuindo-lhe, portanto um caráter de decisão e não de impulso, acreditando ainda que a espiritualidade colabora para o encontro do propósito e do significado da existência humana. Ademais, esse resultado corrobora a compreensão de Lukas (2012) quando concebe que a religiosidade reprimida mitiga a confiança na vida e no seu sentido.

\section{Sentido de Vida e Vitalidade Subjetiva}

A vitalidade associou-se positivamente com a presença de sentido, sugerindo que quanto maior a percepção de um sentido para a vida maior o sentimento de vivacidade e energia pessoal do idoso. Não obstante, torna-se necessário apontar que a magnitude do coeficiente de correlação dessa associação pode ser considerada fraca $(r<0,30$; Cohen, 1988). Compreende-se que a percepção de um sentido pode exercer um importante papel, mas que ela, por si só, não se constitui como o único fator que se relaciona com a vitalidade. Devem-se ter em conta outros aspectos além das questões existenciais, tais como as variáveis psicossociais e psicofísicas, que podem, igualmente, se constituir como fatores relevantes que se relacionam com o construto em questão.

Ademais, deve-se considerar que a própria natureza da medida do sentido na vida não responde a todas as questões acerca desse construto (Steger et al., 2006), o que poderia ter influenciado no baixo índice de correlação. Embora compartilhem pouca variância, considera-se importante tecer alguns comentários sobre essa associação. Segundo a teoria da motivação de Frankl, a vontade de sentido é sempre reforçada por uma percepção de algum propósito na vida, dessa forma a dimensão psíquica seria influenciada positivamente, desencadeando afetos positivos (Frankl, 1969/2011; Lukas, 1992).

Compreende-se que maiores níveis de sentido de vida se constituem como um fator de proteção ao psicofísico (Frankl, 1946/1989), o que 
pode, inequivocamente, influenciar na avaliação da vitalidade subjetiva. Se por outro lado a presença de um sentido pode aumentar a vitalidade, por outro, a sensação de vazio existencial, que é caracterizado pela apatia, pode diminuir a energia e o vigor de uma pessoa idosa.

Sobre a importância de uma tarefa ou missão para o idoso, comenta Frankl (1991): "Essencial e decisivo, sob o aspecto psicológico, é única e exclusivamente que tal atividade desperte no homem, por mais avançada que seja a sua idade, o sentimento de existir para algo ou para alguém" (p. 52). Essa compreensão é corroborada com o estudo de Nix et al. (1999) que sugere que atividades motivadas intrinsecamente aumentam a energia e vitalidade subjetiva. Tendo em conta que a vontade de sentido é uma motivação intrínseca, esta, por sua vez, pode ser compreendida como uma condição relevante na percepção da vitalidade. Assim, torna-se essencial compreender essa última variável no contexto da percepção de sentido na temporalidade (passado, presente e futuro).

\section{Percepção Ontológica do Tempo e Vitalidade Subjetiva}

Conforme sugerem os resultados dessa pesquisa, quanto maior o nível da percepção do passado, do presente e do futuro, maior o escore de vitalidade em idosos. A influência da percepção temporal positiva obteve resultados significativos para com a vitalidade subjetiva, ou seja, quando se tem uma visão positiva do passado, presente e futuro, maior o nível de vitalidade na vida destes idosos. Sobre esta perspectiva Monteiro (2006) enfatiza que a compreensão do tempo alivia o sofrimento humano, uma vez que cada indivíduo é capaz de compreender a temporalidade, por outro lado o sofrimento e angústia da passagem do tempo fazem parte do processo de envelhecimento.

Sobre a temporalidade e transitoriedade, Frankl (1948/2007) a considera essencial na vida humana, na qual o sentimento de gratidão e orgulho pelas realidades contidas no passado fazem parte do monumento da existência em sua totalidade. Lukas (1992) faz referência a um balanço existencial e menciona o passado como conteúdos já realizados e imperecíveis; o presente é compreendido como um conjunto de tarefas a cumprir, que por um lado se esvaem e por outro devem ser cuidadosamente analisada em função daquilo para que se é chamado (dever-ser). No entanto, o futuro apresenta-se como espaço livre, em que o idoso ainda pode arquitetar planos apesar da consciência da brevidade da sua existência. Destarte, o olhar para um futuro permite ao idoso perceber possibilidades a serem realizadas.

Lukas (1990) considera que, de forma geral, por uma suposta falta de sentido no futuro, o indivíduo pode deixar de responder às perguntas que a vida lhe endereça no presente. Em decorrência disso, pode gerar uma depressão noogênica, devido à falta de metas e objetivos na vida, sendo expressa pela sensação de um vazio interior. Assim como Lukas, Frankl dimensiona a vida humana em três tempos: o presente, o passado e o futuro. Segundo ele, onde "o presente é a fronteira entre a não realidade do futuro e a realidade eterna do passado" (Frankl, 1978/2005b, p. 101). O passado é perenizado na morte, nada mais se modifica, pois o ser não possui mais nem a mente, nem o corpo, restando-lhe apenas o seu ser passado. Assim, para a perspectiva do idoso, quando o futuro é compreendido como possibilidades de ser, o seu ser ainda estaria por se constituir, o que geraria, segundo Frankl (1946/2005a), uma tensão saudável.

Desta forma, a finitude pode ser considerada um elemento positivo da condição humana, na medida em que a transitoriedade da existência não pode tirar o seu sentido; ao mesmo tempo, torna-se essencial para a busca de sentido no estágio final do ciclo da vida.

\section{Considerações Finais}

A presente pesquisa permitiu conhecer as relações entre variáveis que anteriormente eram ignotas. Pôde-se compreender melhor quais as relações entre a atitude religiosa e o sentido da vida, assim como a percepção ontológica do tempo com a vitalidade em uma amostra eminentemente composta por idosos. Também teve o mérito de verificar a fatorabilidade de uma me- 
dida de Vitalidade Subjetiva para essa fase do desenvolvimento humano, atentando para que as futuras pesquisas possam averiguar uma análise confirmatória. Ademais, sugere-se que estudos complementares sejam realizados para dar continuidade à compreensão do tema em foco, com o intuito de descobrir novos potencializadores da vitalidade nesse período do ciclo da vida.

Considera-se imperativo que os resultados desse estudo possam nortear a prática de profissionais de saúde, em especial psicólogos que atuam no âmbito de temas pertinentes a um envelhecimento ativo. Conforme Melo, Eulálio, Gouveia e Silva (2013), as intervenções reguladas por meio dos pressupostos da Logoterapia podem ser uma ferramenta adequada para trabalhar a saúde e qualidade de vida em idosos.

Por conseguinte, não apenas a capacidade de retrospecção parece ser importante para a vitalidade, mas também a prospecção (i.e., a capacidade de percepção de sentidos e significados no presente e no futuro). Auxiliar o idoso a ressignificar o seu passado, perceber tarefas no presente e ampliar a perspectiva do futuro, constitui uma forma de promoção da vitalidade na pessoa idosa.

Assim, torna-se importante que o profissional da psicologia exerça um papel significativo na atuação junto aos idosos, seja no âmbito de psicoterapia de grupo ou individual, ou programas preventivos, investigando e ampliando possíveis sentidos no passado, no presente e no futuro por meio de um balanço existencial. Assim, o profissional pode promover novas descobertas de sentido que despertem um aumento na vontade de sentido, inerente ao ser humano e basilar para a sua saúde mental, o que pode ocasionar, por conseguinte, um fortalecimento na vitalidade do idoso.

\section{Referências}

Aquino, T. A. A. (2009). Atitudes e intenções de cometer o suicídio: Seus correlatos existenciais e normativos (Tese de doutorado não publicada). Universidade Federal da Paraíba, João Pessoa, PB, Brasil

Aquino, T. A. A. (2013). Logoterapia e análise existencial: Uma introdução ao pensamento de Viktor Frankl. São Paulo, SP: Paulus.
Aquino, T. A. A., Correia, A. P. M., Marques, A. L. C., Souza, C. G., Freitas, H. C. A., Araújo, I. F., ...Araújo, W. F. (2009). Atitude religiosa e sentido da vida: Um estudo correlacional. Psicologia: Ciência e Profissão, 29, 228-243. Recuperado em http://www.scielo.br/pdf/pcp/v29n2/ v29n2a03.pdf

Aquino, T. A. A., Gouveia, V. V., Silva, S. S., \& Aguiar, A. A. (2013). Escala de Atitudes religiosas, versão expandida (EAR - 20): Evidências de validade. Avaliação Psicológica, 12, 109119. Recuperado em http://pepsic.bvsalud.org/ $\mathrm{pdf} / \mathrm{avp} / \mathrm{v} 12 \mathrm{n} 2 / \mathrm{v} 12 \mathrm{n} 2 \mathrm{a} 02 . \mathrm{pdf}$

Araújo, M. F. M., Almeida, M. I., Cidrack, M. L., Queiroz, H. M. C., Pereira, M. C. S., \& Menescal, Z. L. (2008). O papel da religiosidade na promoção da saúde do idoso. Revista Brasileira em Promoção de Saúde, 21, 201-208. doi: $10.5020 / 18061230$

Ardelt, M. (2003). Effects of religion and purpose in life on elders' subjective well-being and attitudes toward death. Journal of Religious Gerontology, 14, 55-77. doi:10.1300/J078v14n04_04

Ardelt, M., \& Koenig, C. S. (2007). The importance of religious orientation and purpose in life dying well: Evidence from three cases studies. Journal of Religion, Spirituality \& Aging, 19, 61-79. doi:10.1300/J496v19n04_05

Bostic, T. J., Rubio, D. M., \& Hood, M. (2000). A validation of the subjective vitality scale using structural equation modeling. Social Indicators Research, 52, 313-324. Retrieved from http://mres. gmu.edu/pmwiki/uploads/Main/Bostic2000.pdf

Carneiro, L. C. (2009). Religiosidade e qualidade de vida em idosos institucionalizados (Dissertação de mestrado, Programa de Pós Graduação em Ciências das Religiões, Universidade Federal da Paraíba, João Pessoa, PB, Brasil).

Cohen, J. (1988). Statistical power analysis for the behavioral sciences. Hillsdale, NJ: Laurence Erlbaum.

Conselho Nacional de Saúde. (2012). Resolução $n^{\circ}$ 466, de 12 de dezembro de 2012. Brasília, DF: Autor. Recuperado em http://www.conselho. saude.gov.br/web comissoes/conep/index.html

Damásio, B. F. (2013). Sentido de vida e bem-estar subjetivo: Interações com esperança, otimismo, autoeficácia e autoestima em diferentes etapas do ciclo vital (Tese de doutorado, Universidade Federal do Rio Grande do Sul, Porto Alegre, RS, Brasil). 
Dendena, A., Dallazen, C. C., Lyra, L. R., \& Tosi, P. C. S. (2011). Religiosidade e envelhecimento bem sucedido. Unoesc \& Ciência- ACHS, 2, 184-196. Recuperado em http://editora.unoesc. edu.br/index.php/achs/article/view/693/pdf_227

Duarte, Y. A. O., Lebrão, M. L., Tuono, V. L., \& Laurenti, R. (2008). Religiosidade e Envelhecimento: Uma análise do perfil de idosos do município de São Paulo. Revista de Saúde Coletiva, 5, 173-177. Recuperado em http://www. fsp.usp.br/sabe/Artigos/2008_Yeda_Religiosidade_SCol.pdf

Frankl, V. E. (1989). Psicoterapia e sentido da vida (A. M. Castro, Trad.) São Paulo, SP: Quadrantes. (Original publicado em 1946)

Frankl, V. E. (1991). Psicoterapia para todos: Uma psicoterapia coletiva para contrapor-se à neurose coletiva (A. E. Allgayer, Trad.). Petrópolis, RJ: Vozes.

Frankl, V. E. (2005a). Em busca de sentido: Um psicólogo no campo de concentração (12. ed., W. O. Schlupp \& C. C. Aveline, Trads.). Petrópolis, RJ: Vozes. (Original publicado em 1946)

Frankl, V. E. (2005b). Um sentido para a vida. Psicoterapia e Humanismo (V. H. S. Lapenta, Trad.). Aparecida, SP: Ideias \& Letras. (Original publicado em 1978)

Frankl, V. E. (2007). A presença ignorada de Deus (10. ed., W. O. Shlupp \& H. Reinhold, Trads.). Petrópolis, RJ: Vozes. (Original publicado em 1948)

Frankl, V. E. (2011). A vontade de sentido. Fundamentos e aplicações da Logoterapia (I. S. Pereira, Trad.). São Paulo, SP: Paulus. (Original publicado em 1969)

Freitas, A. C. P. (2010). Espiritualidade e sentido de vida na velhice tardia (Dissertação de mestrado, Programa de Pós Graduação em Ciências da Religião, Pontifícia Universidade Católica de Minas Gerais, Belo Horizonte, MG, Brasil).

Goldstein, L. L., \& Sommerhalder, C. (2002). Religiosidade, espiritualidade e significado existencial na vida adulta e velhice. In E. V. Freitas, L. Py, A. L. Neri, F. A. X. Cançado, M. L. Gorzoni, \& S. M. da Rocha (Eds.), Tratado de geriatria e gerontologia (pp. 950-956). Rio de Janeiro, RJ: Guanabara Koogan.

Gouveia, V. V., Milfont, T. L., Gouveia, R. S. V., Medeiros, E. D., Vione, K. C., \& Soares, A. K. S. (2012). Escala de Vitalidade Subjetiva - EVS: Evidências de sua adequação psicomé- trica. Psicologia: Teoria e Pesquisa, 28, 5-13. Recuperado em http://www.scielo.br/pdf/ptp/ v28n1/02.pdf

Guimarães, H. P., \& Avezum, A. (2007). O impacto da espiritualidade na saúde física. Revista de Psiquiatria Clínica, 34, 88-94. Recuperado em http:// www.hcnet.usp.br/ipq/revista/vol34/s1/88.html

Kimble, M. (2009). Logotherapy: End stage perspectives of aging. In A. Batthyany \& J. Levinson (Eds.), Existential psychotherapy: Handbook of Logotherapy and existential analysis (pp. 233244). Phoenix, AZ: Zeig, Tucker \& Theisen.

King, L. A., Hicks, J. L., \& Del Gaiso, A. K. (2006). Positive effect and the experience of meaning in life. Journal of Personality and Social Psychology, 90, 179-196. doi:10.1037/00223514.90.1.179.

Koenig, H., George, L., \& Siegler, I. (1988). The use of religion and other emotion-regulation coping strategies among older adults. Gerontologist, 28, 303-310. doi:10.1093/geront/28.3.303

Leandro-França, C., \& Murta, S. (2014). Prevenção e promoção de saúde mental no envelhecimento: Conceitos e intervenções. Psicologia: Ciência e profissão, 34, 318-329. doi:10.1590/19823703001152013

Lukas, E. (1990). Mentalização e saúde: $A$ arte de viver e Logoterapia (C. A. Pereira, Trad.). Petrópolis, RJ: Vozes.

Lukas, E. (1992). Prevenção psicológica: A prevenção de crises e a proteção do mundo interior do ponto de vista da Logoterapia (C. A. Pereira, Trad.). Petrópolis, RJ: Vozes.

Lukas, E. (2012). Psicoterapia em dignidade: Orientação de vida baseada na busca de sentido de acordo com Viktor E. Frankl (H. Reinhold, Trad.). Ribeirão Preto, SP: Instituto de Educação e Cultura Viktor Frankl.

Mascaro, N. (2006). Longitudinal analysis of the relationship of existencial meaning with depression and hope (Doctoral dissertation). Texas A\&M University, College Station, TX, USA.

Mascaro, N., \& Rosen, D. H. (2008). Assessment of existential meaning and its longitudinal relations with depressive symptoms. Journal of Social and Clinical Psychology, 27, 576-599. doi:10.1521/jscp.2008.27.6.576

Maugans, T. A. (1996). The spiritual history. Archives of Family Medicine, 5, 11-26. doi:10.1001/ archfami.5.1.11 
Melo, R. L. P., Eulálio, M. do C., Gouveia, V. V., \& Silva H. D. M. (2013). O efeito do estresse na qualidade de vida de idosos: O papel moderador do sentido de vida. Psicologia: Reflexão e Crítica, 26, 222-230. Recuperado em http://www. scielo.br/pdf/prc/v26n2/02.pdf

Menezes, K. M. G. (2012). O lazer como expressão de vitalidade na velhice: Uma experiência das atividades desenvolvidas em um centro de convivência de idosos em Fortaleza - CE. Estudos sobre Envelhecimento, 23, 19-32. Recuperado em http://www.sescsp.org.br/online/

Monteiro, D. da M. R. (2006). Encontrando Deus: Viver in transitus. In D. da M. R. Monteiro (Ed.), Espiritualidade e finitude: Aspectos psicológicos (pp. 13-43). São Paulo, SP: Paulus.

Mowat, H., \& O’Neill, M. (2013). Spirituality and ageing: Implications for the care and support of older people. Retrieved from http://www.iriss. org.uk/sites/default/files/iriss-insight-19.pdf

Nix, G. A., Ryan, R. M., Manly, J. B., \& Deci, E. L. (1999). Revitalization through self-regulation: The effects of autonomous and controlled motivation on happiness and vitality. Journal of Experimental Social Psychology, 35, 266-284. doi:10.1006/jesp.1999.1382

Nunnally, J. C. (1991). Teoria psicométrica. México, DF: Trillas.

Oliveira, E. K de S., \& Silva, J. P. (2013). Sentido da vida e envelhecimento: Relação entre os pilares da Logoterapia e bem-estar psicológico. Revista Logos e Existência, 2, 135-146. Retrieved from http://periodicos.ufpb.br/ojs/index.php/le/article/view/16817/10100

Parker, M., Lee Roff, L., Klemmack, D. L., Koenig, H. G., Baker, P., \& Allman, R. M. (2003). Religiosity and mental health in southern, communitydwelling older adults. Aging \& Mental Health, 7, 390-397. doi:10.1080/1360786031000150667

Paschoal, T., \& Tamayo, A. (2008). Construção e validação da Escala de bem-estar no trabalho. Avaliação Psicológica, 7, 11-22. Recuperado em http://pepsic.bvsalud.org/pdf/avp/v7n1/ v7n1a04.pdf

Pasquali, L. (2003). Psicometria: Teoria dos testes na psicologia e na educação. Petrópolis, RJ: Vozes.

Pintos, C. C. G. (1992). O entardecer da existência: Ajuda para o idoso viver feliz. Aparecida, SP: Santuário.
Pintos, C. C. G. (2014). A síndrome William Wilson: Temporalidade, sentido da vida, velhice e suicídio. In O. L. Oliveros \& P. Kroeff (Eds.), Finitude e sentido da vida: A Logoterapia no embate com a tríade trágica (pp. 239-263). Porto Alegre, RS: Evangraf.

Ramovs, J. (2009). The preventive care significance of Frank's Logoterapy for contemporary programs aimed at quality aging and good intergerational relations. In A. Batthyany \& J. Levinson (Eds.), Existential psychotherapy: Handbook of Logotherapy and existential analysis (pp. 245275). Phoenix, AZ: Zeig, Tucker \& Theisen.

Ryan, R. M., \& Deci, E. L. (2001). To be happy or to be self-fulfilled: A review of research on hedonic and eudaimonic well-being. In S. Fiske (Ed.), Annual Review of Psychology (Vol. 52, pp. 141-166). Palo Alto, CA: Annual Reviews.

Ryan, R. M., \& Frederick, C. M. (1997). On energy, personality, and health: Subjective vitality as a dynamic reflection of well-being. Journal of Personality, 65, 529-565. doi:10.1111/j.14676494.1997.tb00326.x

Silva, A. I., \& Alves, V. P. (2007). Envelhecimento: Resiliência e espiritualidade. Diálogos possíveis. Recuperado em http://www.faculdadesocial.edu.br/dialogospossiveis/artigos/10/14.pdf

Steger, M. F., \& Frazier, P. (2005). Meaning in life: One link in the chain from religiousness to wellbeing. Journal of Counseling Psychology, 4, 574-582.

Steger, F. M., Frazier, P., Oishi, S., \& Kaler, M. (2006). The meaning in Life Questionnaire: Assessing the presence of and search for meaning in life. Journal of Counseling Psychology, 53, 80-93. doi:10.1037/0022-0167.53.1.80

Tabachnick, B. G., \& Fidell, L. S. (2001). Using multivariate statistics. Needham Heights, MA: Allyn \& Bacon.

World Health Organization. (2002). Active ageing: A policy framework. Madrid, Spain: Author. Retrieved from http://whqlibdoc.who.int/hq/2002/ who_nmh_nph_02.8.pdf 